winkel drei Seiten, während der ganze Sachverhalt mit dem Bewegungsbegriff in einem einzigen Satz ausgesprochen werden kann. Auch das gleichschenklige Dreieck wird durch zahlreiche Sätze beschrieben, die -- im Hinblick auf den didaktischen Zweck des Buches - alle durch die eine Aussage: „Das gleichschenklige Dreieck hat eine Symmetrieachse" zu ersetzen wären. Anderseits werden Axiome wie das über die Parallelen und das über die Bestimmung einer Ebene Scheinbegründungen unterworfen. Ist somit der Aufbau des Lehrganges nicht einwandfrei, so muß anderseits hervorgehoben werden, daß zahlreiche und zum Teil neue Anwendungen der Elementargeometrie das Buch interessant und für den Unterricht fruchtbar machen, z. B. die Herstellung von Zierfiguren mit Hilfe der einfachsten Konstruktionen, Feldmessungsaufgaben, Zeichnung technischer Objekte u. a. Auch die Herleitung einzelner Konstruktionen weicht von dem gewöhnlichen Verfahren vorteilhaft $a \bar{b}, z$. B. die stetige Teilung.

$F$.

\title{
Lehr- und Übungsbuch für den planimetrischen Unterricht.
} Von C. Block. 3. Teil. Teubner, 1910.

Die Proportionalitäts- and die Flächenlehre wird in der üblichen Anordnnng and Methode behandelt. Das Übungsmaterial ist reichlich. $F^{\text {? }}$.

Lehrbücher der Planimetrie, Stereometrie und Trigonometrie. Von K. Weber. Wolfenbüttel, Julius Zwißler, 1910/11 (3 Hefte).

Die Grundlegung der Planimetrie fabt an sinnlosen Definitionen so ziemlich alles zusammen, was in dieser Art von verschiedenen Lehrbüchern schon geleistet worden ist. Der Text ist vielfach unklar, die Beweise sind schwerfällig, mitunter unvollständig und falsch (z. B. der für den Peripheriewinkelsatz). Für die Stereometrie gilt ähnliches. Die Trigonometrie bringt nichts methodisch neues and gleichfalls recht schwerfällige Beweise.

F.

\section{Lehrbücher der ebenen Geometrie und Trigonometrie und} der Stereometrie. Von Chr. Sehmehl, Gießen, Emil Roth, 1911/12.

Im Aufbau enthalten diese Bücher nichts wesentlich neues, in der Durchführung wird allgemein ein heuristisches Verfahren mit großer Sorgfalt befolgt: Es wird eine Konstruktionsaufgabe gestellt, die nur geringe Anleitung nötig macht, aus dem Ergebnis der Konstruktion wird der Lehrsatz gewonnen und zugleich darauf hingewiesen, wie gewisse Stücke der Figur von anderen abhängen. Diesem Prinzip entspricht es, daß trigonometrische Formeln in erster Linie nicht durch Umformung, sondern auf geometrisch-anschaulichem Wege gewomnen werden, sowie, das der theoretische Teil der Stereometrie erst nach der Lehre von der Schrägrißzeichnung zur Sprache kommt. Wie der Text, so bezeagen anch die Figuren große Sorgfalt. An Einzelheiten wäre die genäherte Berechnong der goniometrischen Funktionen, die Art, wie die Winkelfunktionen von spitzen auf stumpfe Winkel fortgesetzt werden, die astronomischen Schrägrißdarstellungen and manches andere hervorzuheben. Den verhältnismäBig hohen Anforderungen, die das Buch an den Schüler stellt, würde es entsprechen, daß in den Grundlagen der Planimetrie die üblichen "Definitionen" der einfachsten Gebilde unterdrückt und in der Lehre von den Proportionen und vom Flächen- und Rauminhalt der Begriff des Inkommensuraklen schärfer herausgearbeitet würde. 